\title{
Theoretical Aspects of the Electropolymerization of Some Hydroquinonic Derivatives
}

\author{
Volodymyr V. Tkach 1,2,*(D), Marta V. Kushnir ${ }^{1}$, Sílvio C. de Oliveira ${ }^{2}$, Vitalii V. Lystvan ${ }^{3}$, Inna M. \\ Dytynchenko $^{3}$, Adriano O. da Silva ${ }^{4}$, Yüksel Akınay ${ }^{5}$, Olga V. Luganska ${ }^{6}$, Petro I. Yagodynets ${ }^{1}$, Zholt \\ O. Kormosh ${ }^{7}$ \\ 1 Chernivtsi National University, 58000, Kotsyubyns'ky Str. 2, Chernivtsi, Ukraine \\ 2 Universidade Federal de Mato Grosso do Sul, Av. Sen. Felinto. Müller, 1555, C/P. 549, 79074-460, Campo Grande, MS, \\ Brazil \\ Zhytomyr State University, 10000, Velyka Berdychivska Str., 40, Zhytomyr, Ukraine \\ 4 Universidade Federal do Oeste do Pará, Campus Juriti, Rua V. de Souza Andrade, s/n, 68170-000, Juriti, PA,Brazil \\ 5 Yüzüncü yil University of Van, Bardakçı, Yüzüncü Y1l Üniversitesi Kampüsü, 65090 Tuşba, Van, Turkey \\ 6 Zaporizhzhia National University, 69600, Zhukovsky Str. 66, Zaporizhzhia, Ukraine \\ 7 East Ukrainian National University, 43000, Voli Ave., 13, Lutsk, Ukraine \\ * Correspondence: nightwatcher2401@gmail.com; vvt2401@Chnu.edu.ua;
}

Scopus Author ID 55758299100

Received: 5.06.2020; Revised: 3.07.2020; Accepted: 5.07.2020; Published: 9.07.2020

\begin{abstract}
For the first time, the possibility of the electrochemical synthesis and polymerization of some novel modified naphthoquinone compounds has been described. The correspondent mathematical model has been analyzed by means of linear stability theory and bifurcation analysis. It has been shown that the electro organic synthesis may serve as an interesting substitution for Suzuki reaction for the synthesis of ferrocenyl naphthoquinone and its polymer. The oscillatory and monotonic instability, in this case, will be more probable than in the general electropolymerization case.
\end{abstract}

Keywords: naphthoquinones; Suzuki reaction; electro organic synthesis; electropolymerization; stable steady-state.

(C) 2020 by the authors. This article is an open-access article distributed under the terms and conditions of the Creative Commons Attribution (CC BY) license (https://creativecommons.org/licenses/by/4.0/).

\section{Introduction}

Electrochemical polymerization is one of the most used conducting polymer synthesis techniques [1-10]. The electrosynthetized polymers have certain advantages, while compared to the chemically obtained macromolecules. These advantages include: enhanced conductivity, corrosion resistance, facility in the modification, tunability.

The most known conjugated conducting polymers contain alkine chains, carbo- and heterocyclic fragments in their moiety. While electrochemically synthesized, they are doped by a background electrolyte counter-ion or another counter-ion present in the solution. The monomer and electropolymerization techniques may be chosen according to the further use of the resulting polymer.

Recently, the Organic Chemistry group of the Yüzüncü Yil University of Van has obtained a novel ferrocenyl-substituted naphthoquinone derivative [11-12] (Fig. 1). The novel derivative has shown sensitivity to the concentration of hydrogen peroxide and may readily be used as a sensor. Nevertheless, the novel derivative has been obtained by a Suzuki-Miyaura reaction, requiring expensive catalysts and reagents. An electro organic synthesis used to yield 
not only polymers but also their monomers [13-17]. From $1970^{\text {th }}$ on, the monomer electrosynthesis techniques are developed to yield monomers and their polymers.

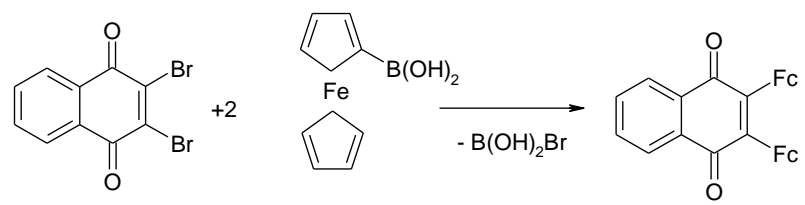

Figure 1. Chemical synthesis of the compound.

Nevertheless, the organic electrooxidation processes (including the electropolymerization) tend to be accompanied by electrochemical instabilities. These instabilities include the oscillatory changes in electrochemical potential and monotonic instabilities [18 - 25] and influence the polymer morphology and conductivity.

Thus, in order to investigate the parameter values, correspondent to the most efficient monomer and polymer formation, like also verify the possibility and probability of the electrochemical instabilities in this system, an a priori theoretical observation of the electrochemical system is necessary.

So, taking into account the above-mentioned statements, the goal of our work will be the theoretical description of the novel ferrocene naphthoquinones electrochemical synthesis as a substitute of the Suzuki reaction, with the further product polymerization.

In order to realize it, we achieve specific objectives as:

- to suggest the most probable mechanism for the sequence of chemical and electrochemical processes in the system;

- to develop the mathematical model, correspondent to the mechanism,

- by analysis of the model, to derive the steady-state stability conditions and oscillatory and monotonic instability requirements;

to compare the behavior of this system with that of the similar ones [25 - 28].

\section{Materials and Methods}

\subsection{System and its modeling.}

In the scheme of the electrochemical synthesis, 1,4-dihydroxynaphthalene is electrochemically oxidized in the presence of 2-chloroferrocene in the medium of carbonate buffer. By this, hydroquinone moiety is oxidized to the quinone one, and the ferrocenyl group integrates the naphthoquinone derivative, forming the monomer (Fig. 2).

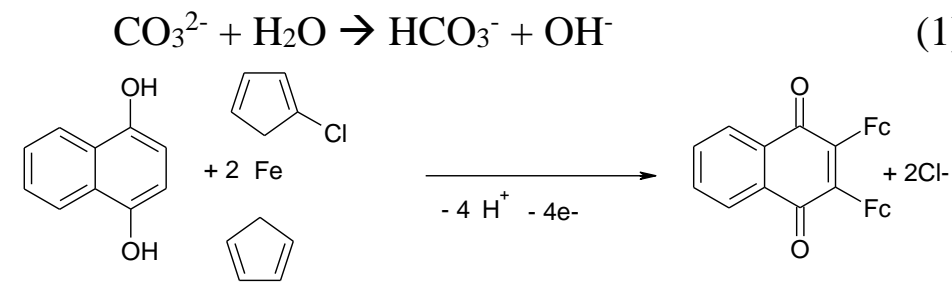

Fig. 2. Monomer electrochemical synthesis

$$
\mathrm{H}^{+}+\mathrm{OH}^{-} \rightarrow \mathrm{H}_{2} \mathrm{O}
$$

The carbonate buffer, added to the reaction medium, prevents the ferrocenyl moieties from acidolytic destruction during both synthesis and electropolymerization (both processes 
expel protons and reduce the $\mathrm{pH}$ values) and also facilitates the hydroquinone to quinone oxidation.

The chain propagation is thus realized by cation-radical formation in the active ferrocenyl moiety and the non-substituted ring of the naphthoquinone fragment, yielding a highly conducting macromolecule, capable of being used in electroanalytic. The polymer morphological and magnetic properties have to be similar to those obtained in [29], but with enhanced conductivity.

Thus, in order to describe the electrochemical behavior of this system in galvanostatic mode, we introduce three variables:

$\mathrm{f}$ - chloroferrocene concentration in the pre-surface layer;

$\mathrm{m}$ - monomer concentration in the pre-surface layer;

$\mathrm{q}$ - anode charge density.

To simplify the modeling, we assume that:

- the background electrolyte is taken in excess so that we can neglect the migration flow and the oxidizing dopant oxidation change;

- the reactor is intensively stirred so that we may neglect the convection flow;

- the pre-surface layer concentration profile is linear, and its thickness is constant, equal to $\delta$.

It is possible to prove that the differential equations'set, describing the system may be described as:

$$
\left\{\begin{array}{c}
\frac{d f}{d t}=\frac{2}{\delta}\left(\frac{D}{\delta}\left(f_{0}-f\right)-r_{E S}\right) \\
\frac{d n}{d t}=\frac{1}{N}\left(r_{E S}-r_{p}\right) \\
\frac{d q}{d t}=i-i_{F}
\end{array}\right.
$$

Herein, $\mathrm{D}$ is the diffusion coefficient, $\mathrm{f}_{0}$ is the ferrocene halide bulk concentration, $\mathrm{N}$ is the monomer maximal surface concentration $i_{F}$ is the Faraday current and the parameters $r$ are the monomer electrosynthesis and polymerization rates, capable to be calculated as:

$$
\begin{aligned}
& r_{E S}=k_{E S} f^{2}(1-n) \exp \left(\frac{4 F \varphi_{0}}{R T}\right) \\
& r_{p}=k_{p} n^{m} \exp \left(\frac{(2 m-2) F \varphi_{0}}{R T}\right)
\end{aligned}
$$

The Faraday current may be calculated as:

$$
i_{F}=4 F r_{E S}+(2 m-2) F r_{p}
$$

Where the parameters $\mathrm{k}$ are correspondent reaction rate constants, $\mathrm{m}$ is the number of the monomer units in the polymer, $\mathrm{F}$ is the Faraday number, $\varphi_{0}$ is the potential slope, related to the zero-charge potential, $\mathrm{R}$ is the universal gas constant, and $\mathrm{T}$ is the absolute temperature of the solution.

Comparing to the potentiostat mode, described for the similar systems in [26], the behavior of this system will be a bit more dynamic. The polymerization kinetics and resulting polymer properties will be affected by this, according to the exposed below.

\section{Results and Discussion}

In order to investigate the galvanostatic electrosynthesis and electropolymerization of the novel ferrocenyl-substituted naphthoquinone, we analyze the equation-set (4) by means of the linear stability theory. The steady-state Jacobian matrix may be exposed like (8): 
In which:

$$
\left(\begin{array}{lll}
a_{11} & a_{12} & a_{13} \\
a_{21} & a_{22} & a_{23} \\
a_{31} & a_{32} & a_{33}
\end{array}\right)
$$

$$
\begin{aligned}
& a_{11}=\frac{2}{\delta}\left(-\frac{D}{\delta}-2 k_{E S} f(1-n) \exp \left(\frac{4 F \varphi_{0}}{R T}\right)\right) \\
& a_{12}=\frac{2}{\delta}\left(k_{E S} f^{2} \exp \left(\frac{4 F \varphi_{0}}{R T}\right)-j k_{E S} f^{2}(1-n) \exp \left(\frac{4 F \varphi_{0}}{R T}\right)\right)(10) \\
& a_{13}=\frac{2}{\delta}\left(-p k_{E S} f^{2}(1-n) \exp \left(\frac{4 F \varphi_{0}}{R T}\right)\right) \\
& a_{21}=\frac{1}{N}\left(2 k_{E S} f(1-n) \exp \left(\frac{4 F \varphi_{0}}{R T}\right)\right) \\
& a_{22}=\frac{1}{N}\left(-k_{E S} f^{2} \exp \left(\frac{4 F \varphi_{0}}{R T}\right)+j k_{E S} f^{2}(1-n) \exp \left(\frac{4 F \varphi_{0}}{R T}\right)-m k_{p} n^{m-1} \exp \left(\frac{(2 m-2) F \varphi_{0}}{R T}\right)-\right. \\
& \left.j k_{p} n^{m} \exp \left(\frac{(2 m-2) F \varphi_{0}}{R T}\right)\right) \\
& a_{23}=\frac{1}{N}\left(p k_{E S} f^{2}(1-n) \exp \left(\frac{4 F \varphi_{0}}{R T}\right)-p k_{p} n^{m} \exp \left(\frac{(2 m-2) F \varphi_{0}}{R T}\right)\right) \\
& a_{31}=-8 F k_{E S} f(1-n) \exp \left(\frac{4 F \varphi_{0}}{R T}\right) \\
& a_{32}=4 F\left(k_{E S} f^{2} \exp \left(\frac{4 F \varphi_{0}}{R T}\right)-j k_{E S} f^{2}(1-n) \exp \left(\frac{4 F \varphi_{0}}{R T}\right)\right)+(2 m- \\
& 2) F\left(m k_{p} n^{m-1} \exp \left(\frac{(2 m-2) F \varphi_{0}}{R T}\right)-j k_{p} n^{m} \exp \left(\frac{(2 m-2) F \varphi_{0}}{R T}\right)\right. \\
& a_{33}=-4 F\left(p k_{E S} f^{2}(1-n) \exp \left(\frac{4 F \varphi_{0}}{R T}\right)\right)-(2 m-2) F\left(p k_{p} n^{m} \exp \left(\frac{(2 m-2) F \varphi_{0}}{R T}\right)\right)(17)
\end{aligned}
$$

In order to derive the steady-state stability requirement, we apply the Routh-Hurwitz criterion to the equation-set (4). Avoiding the cumbersome equations, we introduce new variables, and the Jacobian determinant will be described as:

$$
\frac{2 F}{\delta N}\left|\begin{array}{ccc}
-\kappa-\Lambda_{1} & \Omega & -\Sigma \\
\Lambda_{1} & -\Omega-\Xi & \Sigma-T \\
-4 \Lambda_{1} & 2 \Omega+(m-1) \Xi & -2 \Sigma-(m-1) T
\end{array}\right|
$$

Opening the brackets and applying the Det $\mathrm{J}<0$ conditions, salient from the criterion, we obtain the steady-state stability condition, exposed as (19):

$$
\begin{aligned}
& -\kappa(2 \Xi \Omega+(m-1) \Omega T+\Xi(m-1) \Omega T+2 \Sigma T+\Xi(m-1) \Sigma+(m-1) \Xi T)-\Lambda(2 \Xi \Omega+ \\
& \Xi(m-1) \Omega T+2 \Sigma T+2 \Xi(m-1) \Sigma+(m-1) \Xi T+4(-\Omega T-\Xi \Sigma))<0(19)
\end{aligned}
$$

Describing a more dynamic system, while compared to those observed for potentiostat mode [25]. Taking into account the strong influences of both of the electrochemical processes on double electric layer (DEL) capacitance, ionic force and conductivity, like also the surface resistance, the cyclic changes of which may compromise the steady-state stability, the steadystate stability topological area will be more narrow than for the potentiostat mode.

If in [25], depending on the electrode size and monomer modifier concentration, the system could be only diffusion or only kinetically controlled, in galvanostatic mode, either diffusion or kinetics will influence the electrosynthesis process by equal manner.

Despite the above-mentioned factors, the monomer tends to be formed efficiently, as the topological stability area remains vast. The steady-state is easily stabilized. Nonetheless, the macromolecule morphology will be more dendritic and crystalline than film-shaped.

The monotonic instability for this system is possible. It is defining the margin between stable steady-states and unstable states, and its conditions of appearance will be depicted as (20): 
$-\kappa(2 \Xi \Omega+(m-1) \Omega T+\Xi(m-1) \Omega T+2 \Sigma T+\Xi(m-1) \Sigma+(m-1) \Xi T)-\Lambda(2 \Xi \Omega+$ $\Xi(m-1) \Omega T+2 \Sigma T+2 \Xi(m-1) \Sigma+(m-1) \Xi T+4(-\Omega T-\Xi \Sigma))=0$ (20)

The oscillatory instability for this system, as in the similar ones [25 - 28] is possible. Moreover, it is even more probable than in [25], due to the presence of more than one factor defining the DEL capacitance.

As known, the Hopf bifurcation is realized if the Jacobian matrix main diagonal elements have positive elements, responsible for the positive callback. In these systems, elements, which may be positive are:

$j k_{E S} f^{2}(1-n) \exp \left(\frac{4 F \varphi_{0}}{R T}\right)>0$ if $\mathrm{j}>0$, describing the positive callback, observed during the monomer synthesis. By this, the structure of the surface compound is changed with the enhance of its conductivity, leading to a favorable effect in a double electric layer;

$-j k_{p} n^{m} \exp \left(\frac{(2 m-2) F \varphi_{0}}{R T}\right)>0$ if $\mathrm{j}<0$, describing the analogous effect during the electrochemical polymerization process, which is enhanced by the formation of charged intermediates (cation-radicals).

Other elements, capable of being positive are $-4 F\left(p k_{E S} f^{2}(1-n) \exp \left(\frac{4 F \varphi_{0}}{R T}\right)\right)-$ $(2 m-2) F\left(p k_{p} n^{m} \exp \left(\frac{(2 m-2) F \varphi_{0}}{R T}\right)\right)>0$, if $\mathrm{p}<0$, describing the similar effects of the anode resistance.

We should mention that this model is only suitable for the polymerization in alkaline media, capable of neutralizing the $\mathrm{pH}$ decay, observed during the monomer synthesis and polymerization. If the $\mathrm{pH}$ is neutral or acidic, the ferrocene monomer and polymer suffer partial destruction, influencing the system's behavior and polymer morphology. This aspect will be described in one of our next works.

\section{Conclusions}

From the system with the new ferrocene naphthoquinonic derivative electrochemical synthesis and polymerization, it is possible to conclude that: The electrochemical synthesis of the novel ferrocene derivative of naphthoquinone may be a suitable substitution for the Suzuki reaction; $\mathrm{n}$ galvanostatic mode, the behavior of the system will be more dynamic than in potentiostat mode, due to double electric layer capacitance, ionic force, and conductivity, like surface resistance influences of both electrochemical stages; Also, in galvanostatic mode, the electrosynthesis will be either diffusion or kinetically controlled; The oscillatory instability will be more probable than for potentiostat mode, being caused by DEL influences of both electrochemical stages; Another factor responsible for this type of behavior will be the influence of both electrochemical stages on the anodic resistance.

\section{Funding}

This research received no external funding.

\section{Acknowledgments}

Special aknowledgement to the Yüzüncü Yil University of Van. 


\section{Conflicts of Interest}

\section{The authors declare no conflict of interest.}

\section{References}

1. Yuan, X.; Dragoe, D.; Beaunier, P.; Uribe, D.B.; Ramos, L.; Méndez-Medrano, M.G.; Remita, H. Polypyrrole nanostructures modified with mono- and bimetallic nanoparticles for photocatalytic $\mathrm{H} 2$ generation. Journal of Materials Chemistry A 2020, 8, 268-277, https://doi.org/10.1039/C9TA11088G.

2. Umer, A.; Liaqat, F.; Mahmood, A. MoO3 Nanobelts Embedded Polypyrrole/SIS Copolymer Blends for Improved Electro-Mechanical Dual Applications. Polymers 2020, 12 , https://doi.org/10.3390/polym12020353.

3. Š Setka, M.; Bahos, F.A.; Matatagui, D.; Potoček, M.; Kral, Z.; Drbohlavová, J.; Gràcia, I.; Vallejos, S. Love wave sensors based on gold nanoparticle-modified polypyrrole and their properties to ammonia and ethylene. Sensors and Actuators B: Chemical 2020, 304, https://doi.org/10.1016/j.snb.2019.127337.

4. Nafdez, R.; Kaushik, G,; Tefang, S.; Chel, A. Natural Fiber Reinforced Polymer Composite and Their Tensile Properties. A Review. Letters in NanoBioScience 2020, 9, $1103 \quad-\quad 1110$, https://doi.org/10.332/LIANBS92.11031110.

5. Jung, Y.; Singh, N.; Choi, K.-S. Cathodic Deposition of Polypyrrole Enabling the One-Step Assembly of Metal-Polymer Hybrid Electrodes. Angewandte Chemie International Edition 2009, 48, 8331-8334, https://doi.org/10.1002/anie.200903596.

6. Zhang, L.; Luo, F.; Li, W.; Yan, S.; Chen, Z.; Zhao, R.; Ren, N.; Wu, Y.; Chen, Y.; Zhang, C. Conjugationbroken thiophene-based electropolymerized polymers with well-defined structures: effect of conjugation lengths on electrochromic properties. Physical Chemistry Chemical Physics 2019, 21, 24092-24100, https://doi.org/10.1039/C9CP04308J.

7. Araujo, M.H.D.; Matencio, T.; Donnici, C.L.; Calado, H.D.R. Electrical and spectroelectrochemical investigation of thiophene-based donor-acceptor copolymers with 3,4-ethylenedioxythiophene \% J Polímeros. 2020, 30. https://doi.org/10.1590/0104-1428.03519.

8. Yussuf, A.; Al-Saleh, M.; Al-Enezi, S.; Abraham, G. Synthesis and Characterization of Conductive Polypyrrole: The Influence of the Oxidants and Monomer on the Electrical, Thermal, and Morphological Properties. International Journal of Polymer Science 2018, 2018, https://doi.org/10.1155/2018/4191747.

9. Zembrzuska, D.; Kalecki, J.; Cieplak, M.; Lisowski, W.; Borowicz, P.; Noworyta, K.; Sharma, P.S. Electrochemically initiated co-polymerization of monomers of different oxidation potentials for molecular imprinting of electroactive analyte. Sensors and Actuators B: Chemical 2019, 298, https://doi.org/10.1016/j.snb.2019.126884.

10. Bottari, F.; Moro, G.; Sleegers, N.; Florea, A.; Cowen, T.; Piletsky, S.; van Nuijs, A.L.N.; De Wael, K. Electropolymerized o-Phenylenediamine on Graphite Promoting the Electrochemical Detection of Nafcillin. Electroanalysis 2020, 32, 135-141, https://doi.org/10.1002/elan.201900397.

11. Ertas, N.A.; Kavak, E.; Salman, F.; Kazici, H.C.; Kivrak, H.; Kivrak, A. Synthesis of Ferrocene Based Naphthoquinones and its Application as Novel Non-enzymatic Hydrogen Peroxide. Electroanalysis 2020, 32, 1178-1185, https://doi.org/10.1002/elan.201900715.

12. Kivrak, A.; Zobi, C.; Torlak, Y.; Çamlısoy, Y.; Kuş, M.; Kivrak, H. Synthesis of tetracyanoethylenesubstituted ferrocene and its device properties. Applied Organometallic Chemistry 2018, 32, https://doi.org/4512.10.1002/aoc.4512.

13. Kivrak, A. Development of New Methods for the synthesis of pyrazoles, 4-iodopyrazoles and 1,2,4oxadiazoles. Ph. D. Thesis, Middle East Technical University, 2011

14. Pollok, D.; Waldvogel, S. Electro-organic Synthesis-A. $21^{\text {st }}$ Century Technique. Chem. Sci. 2020, Accepted Manuscript. https://doi.org/10.1039/D0SC01848A.

15. Marta, F.; Monica, O.; Leucio, R.; Achille, I. The Double Role of Ionic Liquids in Electroorganic Synthesis: Green Solvents and Precursors of N-Heterocyclic Carbenes. Current Organic Synthesis 2012, 9, 40-52, https://doi.org/10.2174/157017912798889206.

16. Yuan, Y.; Lei, A. Is electrosynthesis always green and advantageous compared to traditional methods? Nature Communications 2020, 11, https://doi.org/10.1038/s41467-020-14322-z.

17. Ghosh, M.; Shinde, V.S.; Rueping, M. A review of asymmetric synthetic organic electrochemistry and electrocatalysis: concepts, applications, recent developments and future directions. Beilstein Journal of Organic Chemistry 2019, 15, 2710-2746, https://doi.org/10.3762/bjoc.15.264.

18. Das, I.; Goel, N.; Agrawal, N.R.; Gupta, S.K. Growth Patterns of Dendrimers and Electric Potential Oscillations during Electropolymerization of Pyrrole using Mono- and Mixed Surfactants. The Journal of Physical Chemistry B 2010, 114, 12888-12896, https://doi.org/10.1021/jp105183q.

19. Bazzaoui, M.; Bazzaoui, E.A.; Martins, L.; Martins, J.I. Electropolymerization of pyrrole on zinc-leadsilver alloys electrodes in acidic and neutral organic media. Synthetic Metals 2002, 130, 73-83, https://doi.org/10.1016/S0379-6779(02)00101-7. 
20. Das, I.; Goel, N.; Gupta, S.K.; Agrawal, N.R. Electropolymerization of pyrrole: Dendrimers, nano-sized patterns and oscillations in potential in presence of aromatic and aliphatic surfactants. Journal of Electroanalytical Chemistry 2012, 670, 1-10, https://doi.org/10.1016/j.jelechem.2012.01.023.

21. Sazou, D. The dynamical behavior of the electrochemical polymerization of indole on Fe in acetonitrilewater mixtures. Synthetic Metals 2002, 130, 45-54, https://doi.org/10.1016/S0379-6779(02)00110-8.

22. Rahman, S.U.; Ba-Shammakh, M.S. Thermal effects on the process of electropolymerization of pyrrole on mild steel. Synthetic Metals 2004, 140, 207-223, https://doi.org/10.1016/S0379-6779(03)00369-2.

23. Das, I.; Agrawal, N.; Ansari, S.; Gupta, S. Pattern formation and oscillatory electropolymerization of thiophene. Indian Journal of Chemistry - Section A Inorganic, Physical, Theoretical and Analytical Chemistry 2008, 47, 1798-1803.

24. Aoki, K.; Mukoyama, I.; Chen, J. Competition between Polymerization and Dissolution of Poly(3methylthiophene) Films. Russian Journal of Electrochemistry 2004, 40, 280-285, https://doi.org/10.1023/B:RUEL.0000019665.59805.4c.

25. Tkach, V.; Kushnir, M.; Ivanushko, Y.; de Oliveira, S.; Reis, L.; Yagodynets', P.; Kormosh, Z. The theoretical description for the electrochemical synthesis of a squaraine-dye doped conducting polymer. Appl. J. Env. Eng. Sci. 2020, 6, 51-56.

26. Tkach, V.V.; Kushnir, M.V.; Parchenko, V.V.; Aksyonova, I.I.; Odyntsova, V.M.; Luganska, O.V.; Genç, M.; Yagodynets, P.I. The Theoretical Description for Gyromytrin Electrochemical Determination, Assisted by VO(OH) - Reinforced Novel Triazolic Schiff Bases. Appl. J. Env. Eng. Sci. 2019, 5, 334-341.

27. Tkach, V.; de Oliveira, S.; Kushnir, M.; Brazhko, A.; Gala, H.; Luganska, O.; Yagodynets’', P. A descrição matemática do desempenho eletroanalítico do compósito poli(alaranjado da acridina)-oxihidróxido de vanádio na detecção eletrorredutiva da entacapona. 2019, 48, 455-466.

28. Tkach, V.; Kukovs'ka, I.; Ivanushko, Y.; Lukanova, S.; Storoshchuk, N.; de Oliveira, S.; Sluhenska, R.; Tsurkan, M.; Ojani, R.; Yagodynets, P. The Theoretical Evaluation For The Use of Vanadium (III) Oxyhydroxide For The Electrochemical Determination of Benzodiazepines. El-Cezeri Fen ve Mühendislik Dergisi 2018, 5, 292-297, https://doi.org/10.31202/ecjse.370698.

29. Eftekhari, S.; Foroughifar, N.; Khajeh-Amiri, A.; Hallajian, S. Synthesis and Characterization of Polymeric Nanocomposites Based On Poly(Melamine-Paraformaldehyde and Superparamagnetic Silicon Dioxide Loaded Iron (III) Oxide Core-Shell Composite Magnetic Materials. Letters in Applied NanoBioScience, 2020, 9, 914 - 918, https://doi.org/10.332/LIANBS91.914918. 